\title{
ACCURACY OF DOPPLER DETERMINATIONS OF STATION POSITIONS
}

\author{
R. J. ANDER LE \\ Naval Weapons Laboratory, Dahlgren, Virginia, U.S.A.
}

\begin{abstract}
Locations of Doppler satellite observing stations have been revised to obtain a set which is more self-consistent and more consistent with the CIO pole. Residuals of satellite observations for 1970 have been analyzed using the new coordinates to determine mean and standard errors for five days of observations of latitude versus station, time of day, and elevation angle. The accuracy of the determination of latitude is about 4 meters at moderate and high elevation angles. But since more satellite passes occur at lower elevation angles, the accuracy of determination of a component of position based on five days of observation of one satellite is only about 2 meters.
\end{abstract}

\section{Introduction}

Doppler observations of Navy Navigation satellites have been used to determine polar motion since 1969 (Anderle and Beuglass, 1970). Systematic differences of about $1 \mathrm{~m}$ are apparent between the Doppler and astronomic determination of pole positions (Anderle, 1970). These differences still exist after improvements of station coordinates positions and gravity field description (Anderle and Beuglass, 1971). The new coordinate system was shown to produce a consistency of about one meter in station position; but one meter differences are large enough to mask the systematic differences between the Doppler and astronomical results. Therefore, a study of the Doppler observational data was undertaken in a search for biases.

\section{Procedure}

A satellite ephemeris is computed on the basis of each $48 \mathrm{~h}$ of observations made by eighteen base stations. In addition, up to ten mobile stations have contributed data to the orbit determinations at various times. After each orbit computation, the residuals of fit to the final orbit are expressed as two components of error in satellite position for each satellite pass over each station. The tangential error in position is used in the determination of pole position and both components are saved in a magnetic tape library. The tangential error is essentially the latitude error for observations of polar satellites (or negative of the latitude error if the satellite is traveling from North to South). The latitude errors in the library for 1970 were adjusted to the improved NWL 9C coordinate system described by Anderle and Beuglass (1971), were used to determine the pole position in the NWL 9C system, and were then corrected for the difference between the NWL 9C pole position and the nominal pole position used in the original orbit computation. The adjusted errors in the NWL $9 \mathrm{C}$ coordinate system were then analyzed to search for systematic errors. 


\section{Results}

Latitude errors in five day spans were divided into class intervals according to the elevation angle to the satellite. The average and root mean square of the errors for each $5^{\circ}$ of elevation angle were computed for each span and each 90 day span. In computing the mean and rms, each error was weighted inverseley as the square of the mean random error in frequency observation. The random error in frequency was determined in the preprocessing of the data (Anderle, 1965). The latitude error was found to be $4 \mathrm{~m}$ for passes between 45 and $90^{\circ}$ elevation, and increased to about $10 \mathrm{~m}$ for a satellite at $15^{\circ}$ elevation. The errors at the higher elevation are probably about the size of the orbit error (Anderle, et al., 1968), while the errors at the lower elevation angles include degradation of the error due to larger refraction errors and the longer distances to the satellite. The errors for each station were then averaged over five day time spans and the standard error for each group of passes was obtained. In computing these statistics, the elevation angle for each pass was also considered in computing the weight for each error as described by Anderle and Beuglass (1970). The results were computed for passes when the satellite was moving from North to South over each satellite observing station independently from results for South-North passes. The passes in opposite directions are separated by about $12 \mathrm{~h}$ in time for a station near the equator and occur at times when the station is observing a different portion of the satellite orbit; thus biases in the computations are more apparent than when the data are combined. The standard error for each five day mean is about $1.5 \mathrm{~m}$. The mean errors show systematic variations which reach as high as $4 \mathrm{~m}$ for some stations at some times during the year. There were some large gaps in data when a station was not operating during a period of time or when the data were received in the computing center too late for routine processing; however, some individual data points and data for the first 70 days for station 121 are even larger than $4 \mathrm{~m}$. The average latitude errors were computed for all stations. Departures from the mean were found during the early part of the year reflecting the large biases for station 121 . Other variations are also due to individual or small groups of stations rather than consistent variations for all stations. In another search for systematic effects, the latitude errors during each one hour time interval were averaged across 90 day intervals and plotted against hour. While systematic variations were found, they were not consistent for the four time periods. Plots of the results are shown in Naval Weapons Laboratory Report TR-2559, which has the same title as this summary. Copies of the report are available from: Defense Documentation Center, Cameron Station, Alexandria, Virginia 22314 U.S.A.

\section{Discussion}

The results show systematic differences between latitude errors for different stations. In several instances, the difference was due to a 10 or $20 \mathrm{~m}$ error in the latitude of the stations, such as in the case of station 121 for days 1-75 and station 105 for days 
296-325. During these periods, the incorrect nominal latitude of the two stations was large enough to affect the average latitude of all stations and the pole path by one or two meters. On the other hand, the cause of a semi-annual variation in the latitudes of Samoa and Hawaii is unknown. The variations are in phase for the two stations but out of phase for South to North passes as compared to North to South passes. Orbit errors are large enough to produce errors of the observed magnitude, and such errors would be in phase for these two stations because of their relative locations; but there is no reason to expect that such errors would be semi-annual. None of the latitude variations observed for the stations approached the 9 to $16 \mathrm{~m}$ magnitude Pavlov (1971) deduced from time service results observed in 1960 and which he attributed to earthquakes.

\section{Summary}

The latitude of stations making Doppler observations of artificial Earth satellites is determined to a consistency of $4 \mathrm{~m}$ from data from a single satellite passing reasonably near the station. Although at least 20 passes of a single satellite are observed in a five day time span, the standard error for such a set of observations is somewhat over a meter because many of the passes occur in the low elevation angles. Systematic variations are observed in the station latitudes during the year. In some cases the latitude variations are due to use of preliminary station coordinates in the computations and are as large as the observed $1 \mathrm{~m}$ discontinuities in the pole path determined by the Doppler observations and one meter differences between Doppler and astronomical determinations of polar motion. Further analyses of the observational data will be carried out to attempt to determine the cause of other variations.

\section{References}

Anderle, R. J.: 1965, Trans. Am. Geophys. Union 46 (2), 1965.

Anderle, R. J., Malyevac, C. A., and Green, H. L. Jr.: 1969, J. Spacecraft Rockets 6 (8), 951.

Anderle, R. J. and Beuglass, L. K.: 1970, Bull. Geodes. 96.

Anderle, R. J.: 1970, Polar Motion Determinations by U.S. Navy Doppler Satellite Observations', Naval Weapons Laboratory Technical Report 2432, 1970.

Anderle, R. J. and Beuglass, L. K.: 1971, Proceedings of AGU/AIAA Symposium on Application of Artificial Satellite to Geodesy, Washington, D.C.

Pavlov, N. N.: 1971, Soviet Astron. 14, 725.

\section{DISCUSSION}

P. Melchior: When you eliminate one station, does the change in $x$ and $y$ depend upon the geographical situation of the eliminated station.

$R$. J. Anderle: When one eliminates 4 stations situated along the $y$ axis, then we observe big changes in the $y$ coordinate. 